Original research

\title{
Stress and coping strategies among NHS executives in sustainability and transformation partnerships
}

Craig Brown, Head of Provider Portfolio, Imperial College Health Partners, London, UK; Jane Hendy, Chair in Organisation Studies, Brunel Business School, Brunel University London, London, UK; Catherine L. Wang, Professor of Entrepreneurship and Strategy, Brunel Business School, Brunel University London, London, UK

\begin{abstract}
This study investigated stress experienced by NHS executives and their coping strategies following the development of Sustainability and Transformation Partnerships. Lazarus and Folkman's (1984) transactional model of stress and coping was adopted to identify a range of stressors and coping strategies deployed by NHS executives to mitigate stress. An inductive qualitative approach was adopted using interview data, aiming to distinguish potentially harmful stressors from benign stressors and to discuss emotion-based and problem-based coping strategies. Finally, the authors make clear recommendations for NHS England to develop leadership capability regarding inter-role conflict and the development of a regional culture of resilience among system-level executives.
\end{abstract}

Key words

Leadership, NHS, Organisational change, Sustainability and Transformation Partnerships, Transactional model of stress and coping strategies

\section{Introduction}

The NHS faces pressure to deliver increasingly complex healthcare services despite diminishing resources and a predicted funding gap of $£ 30$ billion by 2020/21 (Ham et al, 2017). The NHS Long Term Plan (LTP) aims to implement an accelerated, integrated approach to population health using integrated care systems by April 2021 (NHS England, 2019). However, this culture of 'whole system change' across multiple partners within a region is not new to the NHS. Their 'Five Year Forward View' identified the necessity for large scale integration across traditional NHS boundaries, calling for new models of care on a regional scale (NHS England, 2014). Between 2015 and 2016, Sustainability and Transformation Partnerships (STPs) covering 44 areas were developed (Alderwick et al, 2016) to address the needs of the local health population across Primary Care and NHS Trusts.

It is important to note that, while these STPs provided a vehicle for the delivery of healthcare, they were not statutory bodies, therefore all proposals had to be endorsed and supported by the Boards of the relevant partners before implementation. Executive leaders are thus charged with supporting meso-level system change, as well as being legally accountable for their own organisations within that system.

The capacity of healthcare executives to fulfil these two responsibilities has been questioned (Vize, 2017) and it is unclear whether the creation of STPs has brought additional stress for executives, who already experience significant work-related stress (Blackler, 2006). The personal support for senior NHS leaders has been described as 'woefully inadequate' (Rose, 2015) and the effects of stress on these individuals has implications for recruitment and retention. Indeed, $19 \%$ of NHS CEOs were found to have been in their organisation for less than 12 months, while their median tenure was just under 3 years (Brennan, 2017). This high turnover rate can have a major impact, with several international studies calculating the cost of replacing an executive as being two and a half 
times the outgoing executive salary (Ballantine et al, 2008; Sinnott, 2008; Thrall, 2008). Meanwhile, the leadership gap that opens when a chief executive leaves can have a destabilising effect for an organisation. This may lead to further turnover in other senior leadership positions, as well as creating a vacuum around critical tasks such as strategic planning and resource allocation (Wilson and Stranahan, 2000; Havens et al, 2008).

It is unclear whether the introduction of STPs has exacerbated workplace stress for executives, or whether they have had to alter their coping strategies to accommodate the emergent political landscape of system level changes. The aim of this research was to understand whether and how STPs have caused additional stress for NHS executives, as well as exploring any subsequent coping strategies they have deployed to manage that stress.

\section{The transactional model of stress and coping}

An individual's ability to adapt and cope in a stressful environment can be explored through a number of theoretical models, including the Generalised Adaption Syndrome (GAS) model (Selye, 1976) and Cox's Model of Stress (Cox, 1978). One of the most well-known methods is that of Lazarus and Folkman (1984) (Figure 1). Described as a 'transactional model', it explores the interactions (transactions) between the person and their environment over time through their reappraisal of the situation, making it a dynamic and reflexive process.

Stress is conceptualized in this model as the discrepancy between the perceived demands of a situation and the individual's resources to deal with those demands (Harrop et al, 2006). The primary appraisal process identifies the threat as either irrelevant, harmful or potentially positive, while the secondary appraisal explores the ability of the individual to control or cope with the outcome of the primary appraisal (Lazarus and Folkman, 1984). Coping strategies are a key component of transactional models. Strategies which aim to modify the stressor to minimise its effects are defined as 'problem focused', while strategies aiming to regulate the individual's emotional response to a stressor are defined as 'emotion focused'.

This model is helpful when exploring complex environmental and political landscapes in which individuals face a range of challenges with differing origins and timeframes for delivery, requiring them to clearly evaluate their ability to influence the outcome.

Figure 1. Lazarus and Folkman's (1984) transactional model of stress and coping

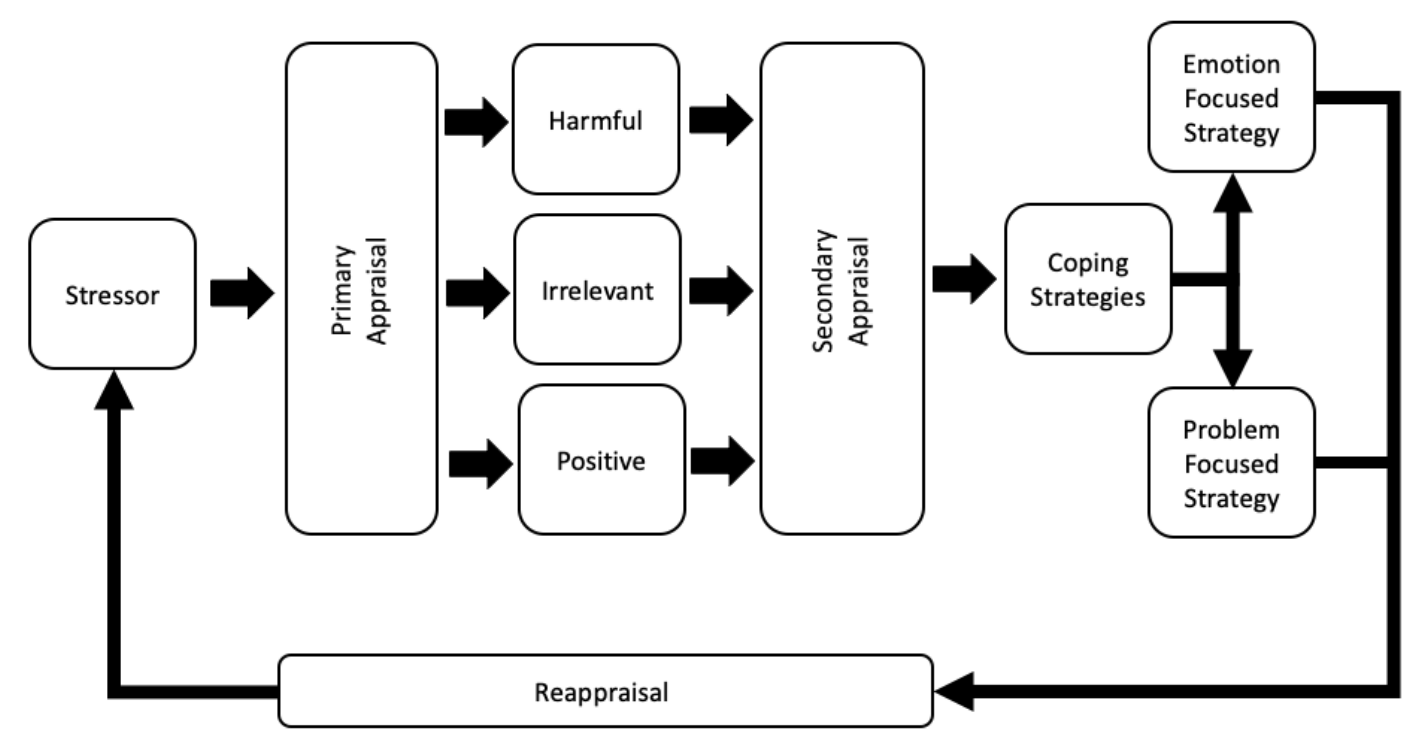




\section{Methods}

An inductive qualitative approach was adopted to explore NHS executives' experiences, opinions and personal reflections regarding the STP. Purposive sampling identified individuals who met the inclusion criteria, which required them to have 'Chief Executive/Officer' or 'Managing Director' in their title, have held in their post for at least 12 months and be part of the same STP region. This gave a total of eight participants (Table 1).

Table 1. Participant contextual information

\begin{tabular}{|l|l|l|l|}
\hline Participant code & $\begin{array}{l}\text { Years in current } \\
\text { position }\end{array}$ & $\begin{array}{l}\text { Years within } \\
\text { Healthcare }\end{array}$ & Clinical Background \\
\hline P1 & 14 & $40+$ & N \\
\hline P2 & 6 & $20+$ & Y \\
\hline P3 & 6 & $20+$ & N \\
\hline P4 & 3.5 & $20+$ & Y \\
\hline P5 & 4 & $20+$ & N \\
\hline P6 & 4 & $40+$ & Y \\
\hline P7 & 3 & $40+$ & Y \\
\hline P8 & 2 & 7 & N \\
\hline
\end{tabular}

Participant interviews contained a number of core descriptive questions, including professional history, daily challenges, engagement with the STP, personal perspectives, and coping and resilience strategies.

All interviews were undertaken between September and December 2018 in a location chosen by the participant. Interviews lasted between 30 and 45 minutes, were captured using a recording device and fully transcribed, verbatim. Ethical approval was granted by the London Research and Development Department of Brunel University.

The Gioia Method was used to structure and code data, construct 'first order concepts' and 'second order themes', and finally to combine these into 'aggregate dimensions' (Gioia et al, 2013). Initial familiarisation with in-vivo data identified participants' language and words to generate first order (participant based) concepts through constant comparison between different extracts (Corbin and Strauss, 1990). Constant handling and re-reading by the researchers developed an intimate knowledge of the data (Pope et al, 2000). First order concepts were organised into a logical sequence, allowing the emergence of second order themes at a higher level of abstraction. Finally, the flow of material was collapsed into aggregate dimensions, representing several second order themes that shared common issues.

The end point of data analysis was achieved when all of the incidents had been classified and no more themes - or, therefore, aggregate dimensions - emerged from the data.

\section{Findings}


Using the Lazarus and Folkman (1984) transactional framework, data was centred on the nature and causes of stress among participants (primary appraisal) and the coping resources and strategies deployed to manage those stressors (secondary appraisal). The emergent aggregate dimensions (shown in Figure 2) represent either potentially harmful or benign stressors. To be classed under the latter category, stressors must only have been deemed harmful if their effects threatened to impact on participants' strategic goals.

Figure 2. Data structure

\section{$1^{\text {st }}$ Order Concepts}

We are under scrutiny from the regulator

- There is a lot of reporting to NHS England and NHS Improvement

- We have to make sure we have suitable staffing

- Pressure to deliver on Accident and Emergency targets

- We have very bad infrastructure and facilities, some are unsafe for patients

- Our backlog maintenance is one of the largest in the country

- I'm worried about a major catastrophic estate failure

- Awarding local tenders for services to services outside of the sector creates distrust and tension among members

- It limits how our sites can be utilised

- There are bits of the STP that I'm engaged with, but it doesn't dictate what we do

- It hasn't amounted to much more than planning

- It's a process without power $\underline{2}^{\text {nd }}$ Order Themes Aggregate/ Overarching Dimension
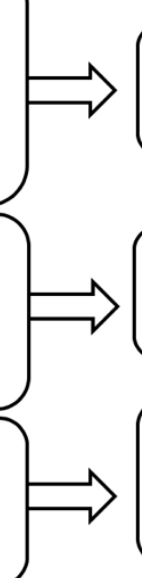

STP creating interrole conflict

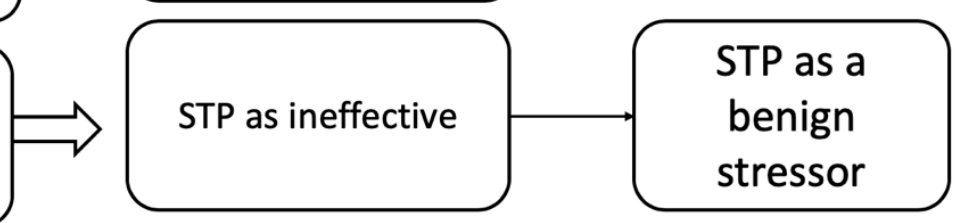

- I believe in our mission

- I remember my core principles and values

- You have got to know your motivation

- I have seen this stuff come round before

- Compliments from patients and locals keeps you going

- I have confidence in my team around me

- If I didn't have a team I would feel less resilient

I speak to my Chair to offload with them 


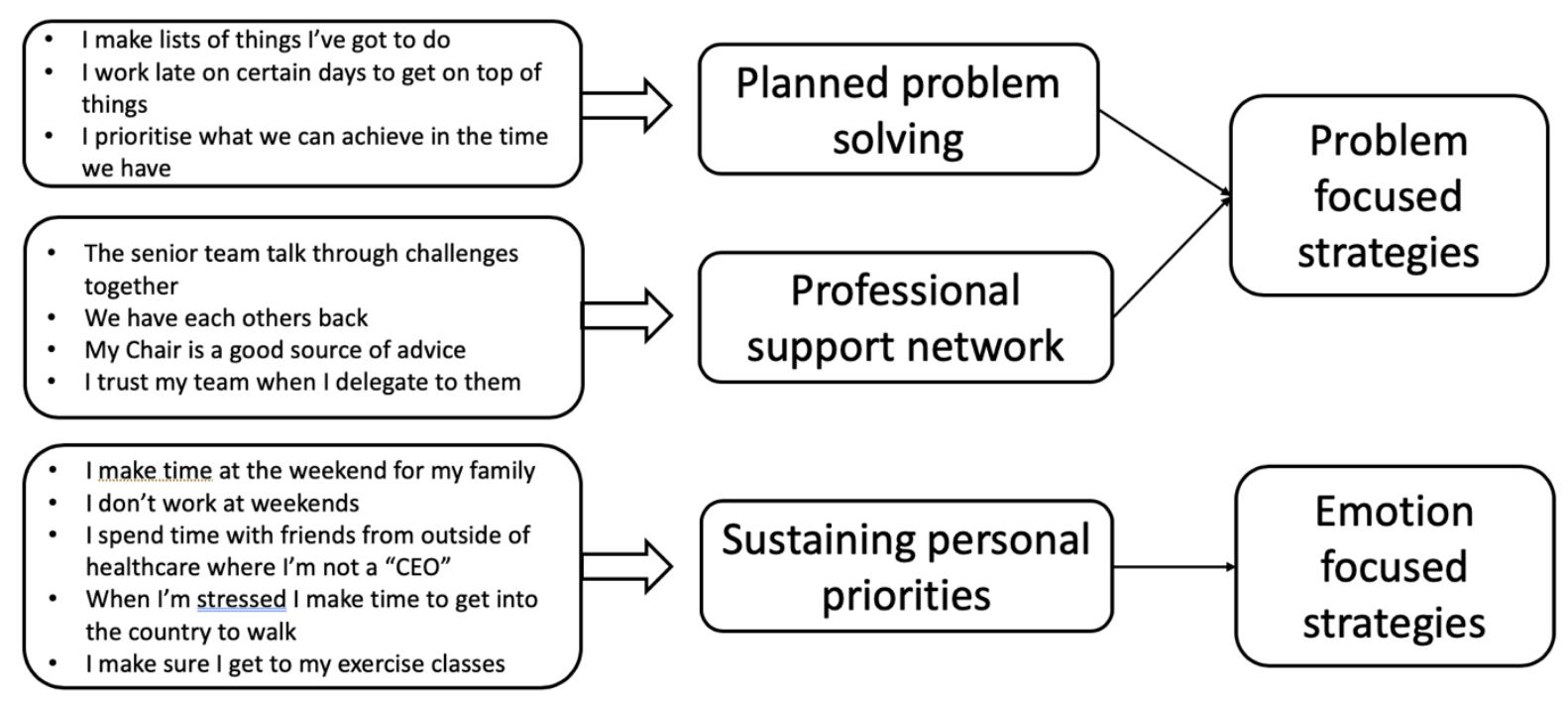

\section{Potentially harmful stressors}

All participants felt that the effects of national regulators, health targets and workforce issues were part of their daily challenges. These produced a stress response, represented in Figure 2 by the second order theme of 'regulatory challenges.' These issues often took considerable time and energy to manage, frequently becoming the most pressing source of stress:

'Day to day, I'm balancing principally A\&E performance, that's the headline one but there's obviously pressure on 18 weeks RTT [referral to treatment] with cancer. So, money, performance and quality then responding to our CQC [Care Quality Commission] report and action plan, that's the three day to day pressures we face,' (P2).

Participants also reported significant stress around the capital estate and sustainability of services. The concepts which fell under the second order theme of 'infrastructure challenges' were suitably different from the 'regulatory challenges' as these stressors did not have short-term or immediate solutions, yet appeared to weigh just as heavily on participants:

'We have very bad design environments to treat and care for patients and in some respect some of them are unsafe.... So that keeps me awake.... We cannot stay here. It's an unsustainable model,' (P1).

The final potentially harmful stressor identified as a second order theme was when the STP created inter-role conflict for individuals. This kind of role conflict is associated with tensions that arise when individuals are required to occupy multiple roles with potentially conflicting expectations. The distrust that these conflicts appeared to facilitate acted as a stressor, especially if the potential consequences would impede the growth of the organisation:

'We're going to award a contract to a bidder that meets our financial requirements that we believe demonstrates best quality and in running an evidence-based process there are going to be winners and losers, and at the start you know, your winners and losers are going to be within your STP footprint,' (P8).

\section{Sustainability and Transformation Partnership as a benign stressor}

Analysis identified the STP itself as a 'benign' stressor, indicating that it was not harmful in certain circumstances, but could not be considered 'irrelevant'. The data analysis demonstrated that some participants lacked a sense of engagement with the STP and perceived that it was not 
particularly effective in delivering system-level change. The only time this differed was when the STP agenda directly threatened participants' organisational strategy, making it a potentially harmful stressor. Otherwise, the participants broadly saw the STP as benign in its effectiveness and capacity to draw their attention.

'I've been around long enough to know that STPs are a flavour of the era and so far, they've been planning. They haven't really amounted to much more than just planning. There's been no execution of any kind that is recognizable.' (P1)

'I would say on a scale of zero to 10, 10 being the most engaged you could be, I would say you'd be lucky if it was six.' (P6)

\section{Coping resources}

Participants identified drawing upon internal core values and meaningfulness as an important way of coping with stress.

'So, I think having a core set of values that you're going to stick to.... having that there is quite important, because in the bad times, you need to go back to "why am doing this?", (P2).

Participants trusted the skills, expertise and capabilities of the broader team to support them. For some, this included the Board and Chair, who were essential to sustaining productive working relationships.

'I think the relationship probably between a chair and a Chief Executive is really important because that's really the only person you've got to unload to.... but they should be there to give you some advice as well or even just some support, ' (P6).

\section{Emotion-focused strategies}

Participants within this study deployed a range of coping strategies, including creating and sustaining environments where they could 'switch off' from work and renew their mental health. Exercise and family environments featured consistently, as well as spending time in the countryside:

I'm a big gym fan, so I go to the gym. I try and get off reasonably sharp in a Friday so I can go to a class, I do quite a lot of classes over the weekend.... exercise I think I would say is my drug of choice and that's really important to me because you have to squeeze that time in somewhere and you've got to have something that makes you not think about work, '(P6).

\section{Problem-focused strategies}

Participants discussed a variety of problem-focused strategies. These included 'planned problem solving', such as the practical application of lists and prioritisation exercises, as well as seeking external support to modify or amend stress.

'I work longer hours, because...well, I form a plan, work through a list,' (P8).

The contribution of the broader team in supporting the participant with discussion and problem solving was a key element of the problem-focused approach. 
'Sitting in a room on my own with the problem or an issue is never going to solve that problem for me. I need to go out and do a "how can you help me?" or "could you do this for me?" and then I can get back on track, '(P4).

\section{Discussion}

Aspects of the STP only emerged as potentially harmful stressors when the STP directly threatened the participants' sense of organisational autonomy. The creation of STPs as regional delivery vehicles, without the power to implement or enforce change, meant that these executives had to balance two potentially conflicting environments. While acting as a leader within the STP, they also occupied the role of CEO or MD in a specific organisation, leading to 'inter-role conflict' (Pandey and Kumar, 1997).

Inter-role conflict has been found to create stress by requiring individuals to fulfil conflicting expectations (Mohr and Puck, 2007). This was evident in the tension and distrust generated by the commissioning arrangements described by participants during the present study. Clinical Commissioning Groups (CCGs) must demonstrate effective fiscal management to NHS regulators regarding the delivery of care for their local population. In order to do this, CCGs are obliged to explore alternate healthcare providers both within their STP and among commercial providers as part of the tendering process.

This process, though necessary, clearly was a source of stress for both the current STP healthcare providers (hospitals) and the CCGs who were required to undertake the process. This can be seen through the comments provided by both the Commissioners and Providers with the same STP:

"And at the start, you know, your winners and losers are going to be within your STP footprint well certainly if your incumbent providers chose not to bid then they're only ever going to be losers and they're within your footprint....so when you're trying to navigate that process and you automatically have winners and losers because of decisions made by providers, .... It's a huge amount of stress because you're managing those stakeholders to try to help you get to the end outcome. That's not popular with them because it causes them a financial headache. "(P8)

"Okay, what's happening and what does this mean for my organisation? And can I either control the anxiety I have that my organisation's losing out in some way that's going to wound it in a sense or am I going to be the bigger person and say, "Well, if it's for the greater good, I'm happy to do that." It takes a very strong person to do that, to say, "We will put our head in the block. And for the greater good of the rest of you....I'm not saying you can't get that happening but you don't get it happening too often." (P6)

This commissioner specific stress has not been explored in any of the current published literature, but may provide further insights towards improving system-wide relationships across regional healthcare areas.

The level of engagement with the STP was inconsistent among participants. Alongside ambiguity in the decision-making process, this produced varied commitment to the STP. This links closely with research on joint ventures, which found that factionalism lead to poor decision-making and lower commitment to decisions, especially when a lack of cultural identity independent of the parent companies existed ( $\mathrm{Li}$ et al, 2002). If we were to consider the STP as an informal 'joint 
venture' across the region, then the absence of a known STP culture and identity may have impeded commitment to system-wide change programmes.

Finally, this study demonstrated that a stress and coping framework can be effectively applied to data of this type. The Lazarus and Folkman model (1984) supported the description of both the perceptions and 'primary appraisal' of stress undertaken by participants, as well as the coping resources and strategies they used. The data indicates that participants drew on their own internal resources and their broader organisational teams, including the Board, for support. However, none of the participants mentioned a sense of peer support across organisational boundaries, implying a lack of support and sense of team identity across the STP.

The STP creates an opportunity to create a stronger sense of cohesion and joint culture with the implementation of a specifically designed coaching programme undertaken at an STP/system level. This may facilitate an improved sense of team identity, possibly leading to improved levels of engagement and mutual support irrespective of organisational boundaries.

\section{Conclusion}

The findings of this study align with existing work around sources of stress among NHS executives and the subsequent coping strategies used by these individuals. However, the present study also demonstrated the tension and stress created by inter-role conflict, manifested in concerns regarding the regional-wide approach to capital infrastructure and the tensions created in commissioning structures.

The published LTP clearly sets out the intention for integrated care systems to supersede the initial STP framework in order to deliver change programmes on a regional level. Within the plan NHS England intends to seek legislative changes to ensure that regional healthcare planning is robust and works towards wider regional objectives rather than those of individual organisations. However, individual leaders will still be expected to facilitate these changes. For this to be successful, the skills and capabilities required of emergent and existing leaders in these complex, boundary-spanning roles must be understood.

The new structures will need to develop an environment and culture that enables individuals to consider their regional leadership body as a team which supports their coping strategies and can function as a collective coping resource. Executive coaching techniques have been found to be effective in developing these cultures and a sense of resilience, however, these studies have been limited in scale, therefore future research requires wider implementation.

\section{Key points:}

- The Lazarus and Folkman transactional model of stress and coping can be applied to explore the impact of stress among NHS executives

- NHS executives can experience inter-role conflict when acting as part of a larger system-level change programme

- Managing Directors within commissioning structures may experience professional stress but this has not been formally quantified

- There are opportunities to develop system level leadership coaching programmes in light of the emerging legal entities of the integrated care systems. 
I would like to acknowledge Emma Balfe, Dr Leyla Osman and Susan Brown.

\section{References}

Alderwick H, Dunn P, McKenna H, Walsh N, Ham C. Sustainability and transformation plans in the NHS: how are they being developed in practice? London: The Kings Fund; 2016.

Ballantine J, Forker J, Greenwood M. The Governance of CEO incentives in English NHS Hospital Trusts. Financ Account Manag. 2008;24(4):385-410. https://doi.org/10.1111/j.14680408.2008.00459.x

Blackler F. Chief executives and the modernization of the English National Health Service. Leadership. 2006;2(1):5-30. https://doi.org/10.1177/1742715006060651

Brennan S. Trust chiefs staying a little longer in post - but 'need more support'. 2017. https://www.hsj.co.uk/workforce/trust-chiefs-staying-a-little-longer-in-post-but-need-moresupport/7020146.article (accessed 25 July 2018)

Corbin JM, Strauss A. Grounded theory research: procedures, canons, and evaluative criteria. Qual Sociol. 1990;13(1):3-21. https://doi.org/10.1007/BF00988593

Cox T. Stress. London: Macmillan; 1978.

Gioia DA, Corley KG, Hamilton AL. Seeking qualitative rigor in inductive research. Organ Res Meth. 2013;16(1):15-31. https://doi.org/10.1177/1094428112452151

Ham C, Alderwick H, Dunn P, Helen M. Delivering sustainability and transformation plans: from ambitious proposals to credible plans. London: The Kings Fund; 2017.

Harrop E, Addis S, Elliott E, Williams G. Resilience, coping and salutogenic approaches to maintaining and generating health: a review. Cardiff Institute of Society, Health and Ethics, 2006.

Havens DS, Thompson PA, Jones CB. Chief nursing officer turnover: chief nursing officers and healthcare recruiters tell their stories. JONA: The Journal of Nursing Administration. 2008;38(12):516-525. https://doi.org/10.1097/NNA.0b013e31818ebf32

Lazarus RS, Folkman S. Stress, appraisal, and coping. New York: Springer; 1984.

Li J, Xin K, Pillutla M. Multi-cultural leadership teams and organizational identification in international joint ventures. Int J Hum Res Manag. 2002;13(2):320-337. https://doi.org/10.1080/09585190110103043

Mohr AT, Puck JF. Role conflict, general manager job satisfaction and stress and the performance of IJVs. Eur Manag J. 2007;25(1):25-35. https://doi.org/10.1016/j.emj.2006.11.003

NHS England, Care Quality Commission, Health Education England, Monitor, NHS Trust Development Authority and Public Health England. NHS five year forward view. London: NHS; 2014.

NHS England. The NHS long term plan. 2019. England. https://www.longtermplan.nhs.uk (accessed: 10 January 2019) 
Pandey S, Kumar ES. Development of a measure of role conflict. Int J Confl Manag. 1997;8(3):187215. https://doi.org/10.1108/eb022795

Pope C, Ziebland S, Mays N. Analysing qualitative data. BMJ. 2000;320(7227):114-116. https://doi.org/10.1136/bmj.320.7227.114

Rose L. Better leadership for tomorrow, NHS leadership review. London: Department of Health; 2015.

Selye H. Stress in health and disease. Boston (MA): Butterworths; 1976.

Sinnott DJ. Leadership turnover: the health care crisis nobody talks about. Chicago (IL): Health Forum; 2008.

Thrall TH. Finding your next CEO. Chicago (IL): Health Forum; 2008.

Vize R. Swimming together or sinking alone: health, care and the art of systems leadership. Institute of Healthcare Management, 2017.

Wilson CN, Stranahan H. Organizational characteristics associated with hospital CEO turnover. J Healthcare Manag. 2000;45(6):395-404. https://doi.org/10.1097/00115514-200011000-00010 